\title{
ALFABETIZACIÓN GENÉTICA Y ADAPTACIÓN PSICOLÓGICA EN ADOLESCENTES CON
} ENFERMEDADES GENÉTICAS

\author{
GENETIC LITERACY AND PSYCHOLOGICAL ADAPTATION IN ADOLESCENTS WITH GENETIC \\ DISEASES
}

\section{ALFABETIZAÇÃO GENÉTICA E ADAPTAÇÃO PSICOLÓGICA EM ADOLESCENTES COM DOENÇAS GENÉTICAS}

\author{
Javiera Ortega ${ }^{1,2,5}$, Natalia Vázquez ${ }^{2,3}$, Claudia Arberas ${ }^{4}$
}

\begin{abstract}
Tener una enfermedad o condición genética representa un desafío para los adolescentes, poniendo en riesgo su salud mental. En el estudio se buscó, por un lado, encontrar en que medida adolescentes argentinos con enfermedades genéticas aceptaron y se adaptaron a su condición. Por otro lado, se buscó describir que conocimientos sobre los procesos genéticos involucrados en su condición tenían estos adolescentes. Por último, se analizó si había una relación entre estos conocimientos y la adaptación psicológica. Los resultados mostraron que, si bien estos adolescentes no conocían mucho sobre genética, si se encontraban adaptados a su condición.
\end{abstract}

\section{Conceptos clave:}

Que se sabe sobre el tema:

-La población de adolescentes diagnosticados con enfermedades genéticas constituye un grupo de "riesgo" desde la perspectiva de la psicología de la salud

-Es necesario un abordaje psicoeducativo para acompañar las vivencias de los adolescentes.

-La adaptación psicológica a la enfermedad y la alfabetización genética resultan variables claves para comprender la situación que atraviesan estos adolescentes.

\section{Que aporta este trabajo:}

-No se han identificado estudios a nivel nacional que aborden esta temática en población adolescente.

-Este estudio muestra resultados sobre una muestra de 68 adolescentes que acuden al servicio de genética médica.

-En el estudio se observaron buenos niveles de adaptación psicológica en los adolescentes.

-Solo un $39,7 \%$ de los participantes presentaban un nivel adecuado de alfabetización genética.

- No se encontraron relaciones estadísticamente significativas entre los niveles de adaptación psicológica y los niveles de alfabetización genética. -La alfabetización genética de los adolescentes parecería relacionarse con el nivel de escolaridad de los adolescentes y de sus madres.

1- Consejo Nacional de Investigaciones Científicas y Técnicas (CONICET)

2- Centro de Investigación de Psicología y Psicopedagogía (CIPP)-Facultad de Psicología y Psicopedagogía, Universidad Católica Argentina

3- Fundación de Psicología Aplicada a Enfermedades Huérfanas (Fupaeh)

4- Servicio de Genética Médica - Hospital de Niños Ricardo H. Gutiérrez

5- E-mail de contacto: javiera_ortega@uca.edu.ar

Recibido: 2019-09-24 Aceptado: 2020-01-10

DOI: http://dx.doi.org/10.31053/1853.0605.v78.n1.25498

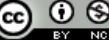

(C) Universidad Nacional de Córdoba

\section{Resumen:}

Introducción: La convivencia con una condición genética es compleja y puede resultar limitante para el adolescente. Esta situación constituye un estresor y un factor de riesgo para la salud mental tanto del paciente como de su familia. Objetivo: Estudiar los niveles de adaptación psicológica y alfabetización genética en adolescentes con enfermedades genéticas, residentes en Argentina. Métodos: Alcance descriptivo correlacional, de diseño cuantitativo y corte transversal. El protocolo administrado consistió en un consentimiento informado, un cuestionario de datos socio-demográficos, y los instrumentos Genetic Health Literacy Screening Tool (REAL G) y Psychological Adaptation Scale (PAS). El muestreo fue intencional y la muestra fue de 68 casos. Resultados: EI REAL G indicó bajos niveles de de alfabetización genética en todos sus componentes (decodificación, familiaridad, comprensión, aritmética). En cuanto a la adaptación psicológica, se encontró una $M=4,02(D E=0.8)$ para la escala general y puntuaciones elevadas para todas las dimensiones. Conclusión: Los adolescentes diagnosticados con enfermedades genéticas en Argentina poseen un buen nivel de adaptación psicológica a su enfermedad, a pesar de tener bajos niveles de alfabetización genética.

Palabras clave: asesoramiento genético; alfabetización en salud; adaptación psicológica; adolescente.

\section{Abstract:}

Introduction: Living with a genetic condition is complex and may be limiting for the adolescent. This situation is considered a stress factor and risk factor for the mental health of both the patient and his family. Objective: To study the levels of psychological adaptation and genetic literacy in adolescents with genetic diseases residing in Argentina. Methods: Correlational and quantitative design and cross sectional scope. The protocol consisted of an informed consent, a sociodemographic data questionnaire, and the Genetic Health Literacy Screening Tool (REAL G) and Psychological Adaptation Scale (PAS) instruments. The sampling was intentional and composed by 68 cases. Results: The REAL $G$ indicated low levels of genetic literacy in all its components (decoding, familiarity, comprehension, arithmetic). Regarding psychological adaptation, an $\mathrm{M}=4.02$ $(S D=0.8)$ was found for the general scale and high scores for all the dimensions. Conclusion: Adolescents diagnosed with genetic diseases in Argentina have a good level of psychological adaptation to their disease, despite having low levels of genetic literacy.

Keywords: genetic counseling; health literacy; adaptation psychological; adolescent.

\section{Resumo}

Introdução: Viver com uma condição genética é complexo e pode ser limitante para o adolescente. Essa situação é considerada um fator de estresse e fator de risco para a saúde mental tanto do paciente quanto de sua família. Objetivo: Estudar os níveis de adaptação psicológica e alfabetização genética em adolescentes com doenças genéticas residentes na Argentina. Métodos: Desenho correlacional e quantitativo e escopo transversal. O protocolo consistiu de um termo de consentimento livre e esclarecido, um questionário de dados sociodemográficos e os instrumentos da Ferramenta de Seleção de Alfabetização em Saúde Genética (REAL G) e da Escala de Adaptação Psicológica (PAS). A amostragem foi intencional e composta por 68 casos. Resultados: O REAL G indicou baixos níveis de alfabetização genética em todos os seus componentes (decodificação, familiaridade, compreensão, aritmética). Em relação à adaptação psicológica, encontrou-se $M=4,02(\mathrm{DP}=0,8)$ para a escala geral e altos escores para todas as dimensões. Conclusão: Adolescentes diagnosticados com doenças genéticas na Argentina apresentam bom nível de adaptação psicológica à doença, apesar de apresentarem baixo nível de alfabetização genética. 


\section{Introducción}

La convivencia con una enfermedad o condición genética es compleja ${ }^{1}$. Diferentes estudios han demostrado que tanto los pacientes, como sus familias se encuentran expuestos a elevados niveles de estrés, ansiedad y depresión ${ }^{2}$.

Al tratarse de condiciones crónicas, requieren de controles médicos constantes, y de tratamientos casi siempre exigentes y demandantes. Algunas de estas condiciones son hereditarias, por lo que involucran a todo el grupo familiar. Un grupo particular de ellas son las llamadas "enfermedades raras" (por su denominación en inglés) o en nuestro medio denominadas "Enfermedades Poco Frecuentes (EPF)", debido a la baja prevalencia de las mismas, 1 cada 2 mil personas ${ }^{3}$. Estas enfermedades, comprenden un cúmulo de patologías muy diferentes, comprenden variadas entidades clínicas y comparten características comunes como la alta complejidad etiológica y diagnóstica, la cronicidad de la enfermedad, el carácter hereditario de más del $80 \%$ de estas, su inicio en ocasiones en la edad pediátrica, cierto grado de discapacidad implicado, y el requerimiento de un manejo transdisciplinario de la condición. Este desconocimiento de la enfermedad por parte del personal de salud, como de la población en general, lleva muchas veces a los pacientes y familiares de éstos a enfrentar la estigmatización de la enfermedad ${ }^{1,4}$. A su vez, el diagnóstico de estas enfermedades trae consigo cierta incertidumbre sobre la evolución y pronóstico de la enfermedad ${ }^{4}$.

Este trabajo tiene como propósito estudiar la experiencia de los adolescentes que asisten a un servicio de Genética Médica del Hospital de niños "Dr. Ricardo Gutiérrez", en Buenos Aires, Argentina, que han logrado tener un diagnóstico de un síndrome genético, una malformación congénita o se encuentran aún en estudio por retraso madurativo leve.

Puede decirse que el diagnóstico de una condición genética representa un factor de riesgo importante para padecer estrés. Si bien muchas familias pueden manejarlo adecuadamente, esa posibilidad no se da en todos los casos $^{5}$.

Distintos autores plantean la importancia de incorporar modelos teóricos de la psicología para atender las necesidades psicológicas de estas familias. El asesoramiento genético, entendido como proceso psicoeducativo, favorece la comprensión de información básica sobre la genética que le permita a la persona tomar decisiones apropiadas con respecto a su salud. Algunos autores lo definen como un proceso psicoterapéutico limitado en el tiempo y centrado en la comunicación de la información genética, favoreciendo la adaptación psicológica a la condición genética o a su riesgo. El cuán bien la persona pueda manejar el estrés determinará cuán bien se adapte a la condición en el tiempo ${ }^{5-6}$.

Más allá de que existe un gran acuerdo entre muchos autores sobre la importancia de este modelo psico-educativo para el abordaje de las enfermedades genéticas, en Latinoamérica y especialmente en Argentina no es un área desarrollada aún en forma sistemática. Un primer paso para iniciar este proceso es contar con información válida y confiable sobre cómo se encuentra la población de interés. Con este propósito la presente investigación buscó estudiar una población particular como adolescentes que acuden a un asesoramiento genético en búsqueda de un diagnóstico.
Antecedentes en población adolescente

La adolescencia, en el desarrollo normal, es una etapa de cambios y movilización, en la que la principal característica suele ser la búsqueda de independencia y la importancia de su grupo de pares ${ }^{7}$. El padecer una enfermedad genética puede resultar limitante para el adolescente. Por un lado, los constantes controles médicos y tratamientos demandantes frecuentemente generan estados de estrés y ansiedad en quien padece la enfermedad ${ }^{8}$. A su vez, estos adolescentes parecen presentar mayor vulnerabilidad percibida, además de una baja autoestima ${ }^{9}$. De igual forma, cobra gran importancia la preocupación en cuanto a algunos aspectos, no menores, como la imagen corporal, las relaciones sociales y las relaciones sexuales ${ }^{10}$.

Por otra parte, son también cotidianos los sentimientos de tristeza, miedo y enojo. Los problemas comportamentales, tales como problemas de sueño, dificultades de aprendizaje y déficits en habilidades sociales se ven también asociados en muchas condiciones genéticas. Estos pacientes suelen tener menor estabilidad emocional, estar menos abiertos a experiencias nuevas, ser menos activos y extrovertidos y mostrar mayor dependencia e irritabilidad ${ }^{11-12}$.

A pesar de los miedos y preocupaciones constantes, los estudios revisados parecen coincidir en que los adolescentes aspiran a tener una vida normal, con relaciones románticas y de amistad y un desarrollo educacional y laboral igual que sus pares ${ }^{13-14}$.De ahí que la interacción con pares y la participación en actividades grupales tome gran importancia en esta etapa ${ }^{14}$.

Si bien estos antecedentes plantean el panorama de la experiencia de convivir con una enfermedad genética en la adolescencia, poco se sabe sobre lo que adolescentes conocen de genética o sobre el grado de adaptación psicológica a su diagnóstico genético.

\section{$\underline{\text { Alfabetización en genética }}$}

La alfabetización en genética es fundamental para comprender la participación efectiva en las decisiones sobre temas genéticos, que favorezcan el bienestar personal. Por alfabetización genética se entiende al grado en que las personas pueden obtener, procesar y entender información básica sobre la genética que le permitan tomar decisiones apropiadas con respecto a su salud. Este concepto comprende el dominio de cuatro componentes principales: reconocimiento, familiaridad, comprensión y aritmética ${ }^{15-18}$. Parte del malestar psicológico que conlleva el diagnostico de una enfermedad genética parecería estar relacionado a la falta de comprensión y conocimiento que existe sobre la naturaleza de la enfermedad ${ }^{1}$.

Estudios anteriores realizados en pacientes adultos o en cuidadores de personas con enfermedades con componentes genéticos, han encontrado que los niveles de alfabetización genética suelen variar. En un estudio realizado con adultos con historia familiar de cáncer, se encontró que los pacientes encontraban confusas las relaciones entre cromosomas, genes y ADN, a pesar de tener conocimientos generales sobre genética ${ }^{19}$. Otro trabajo realizado en cuidadores de niños con hemofilia encontró que éstos tenían poco conocimiento sobre la enfermedad y desconocían los términos usados por los médicos ${ }^{20}$. En una evaluación en pacientes adultos con enfermedades crónicas, se encontró que un nivel elevado de alfabetización genética parecería relacionarse a niveles 
educativos altos, mientras que los niveles de alfabetización podrían disminuir con la edad ${ }^{21}$. Distintos estudios realizados con padres de niños con malformaciones cardiacas congénitas, coinciden en que los padres suelen identificar bien la malformación cardiaca de sus hijos y tienen conocimientos elevados en cuanto a la interacción entre los genes y los factores ambientales, pero presentan mayores dificultades para explicar el componente hereditario de ésta, así como el funcionamiento y estructura de genes y cromosomas $^{22-23}$. No se han encontrado reportes o estudios sobre alfabetización genética en adolescentes que acuden a un servicio de genética médica.

\section{Adaptación psicológica}

El concepto de adaptación psicológica, es decir, el grado en que una persona se encuentra adaptada a una condición genética, es considerado como un constructo dinámico y multidimensional capaz de abarcar tanto los aspectos emocionales como cognitivos del afrontamiento ${ }^{6,24}$. Este concepto incluye las dimensiones de eficacia de afrontamiento, autoestima, integración social y bienestar espiritual / existencial.

Estudios previos sugieren que la severidad de la enfermedad no predice el proceso de adaptación, más bien parecería que en este proceso intervienen mediadores dentro de los que se encuentran la capacidad y el deseo de acceder a los recursos cognitivos necesarios para adaptarse ${ }^{5}$.

En cuanto a los niveles de adaptación psicológica en personas con enfermedades genéticas, se ha encontrado que la mayoría de los pacientes y familiares de éstos logran manejar el estrés que la enfermedad provoca y muestran una gran capacidad de resiliencia ${ }^{5,25}$. Sin embargo, pareciera ser que no todas las familias logran esta adaptación psicológica a la enfermedad.

Algunos estudios plantean que los pacientes no cuentan con las herramientas de afrontamiento necesarias para manejar el estrés que la enfermedad provoca. Además se ha demostrado que los pacientes diagnosticados con enfermedades genéticas presentan menores niveles de adaptación psicológica que pacientes con diagnósticos de otro tipo de enfermedades ${ }^{26}$. Contrario a éstos, un estudio realizado con adultos con fibrosis quística y espina bífida encontró que los pacientes adultos contaban con estrategias de afrontamiento efectivas que los llevaban a tener mejores relaciones interpersonales y funcionamiento social en general $^{27}$.

Un trabajo realizado con adolescentes con Síndrome de Klinefelter encontró niveles elevados de adaptación psicológica en estos adolescentes y plantea que la adaptación a la enfermedad estaría relacionada con el uso de estrategias de afrontamiento centradas en el problema ${ }^{28}$. De igual forma, el apoyo social parece interferir de forma positiva en los niveles de adaptación psicológica tanto de los niños como de sus padres ${ }^{25}$.

La población de adolescentes diagnosticados con enfermedades genéticas constituye un grupo de "riesgo" desde la perspectiva de la psicología de la salud y representan un área de vacancia, considerando la producción científica de Argentina. En este contexto, esta investigación se propuso investigar los niveles de alfabetización genética y el grado de adaptación psicológica alcanzado por esta población de adolescentes.

\section{Métodos}

Se trata de un estudio de diseño no experimental, correlacional, de corte transversal.

\section{Población y procedimientos para obtener la muestra}

La selección de la muestra fue no probabilística, por conveniencia. El universo de estudio fueron adolescentes y preadolescentes que acudían a la Sección de Genética Médica del Hospital de niños "Dr. Ricardo Gutiérrez", residentes en Argentina. Quedaron excluidos del estudio aquellos cuadros genéticos que tengan asociados un retraso severo, dado que no podrían responder a los cuestionarios. Se llegó a una muestra de 68 adolescentes y preadolescentes, de entre 9 a 19 años con una edad media de 14.07 años (DE= 2.48). En cuanto al sexo de los participantes, el $55.9 \%$ de la muestra eran varones y el $44,1 \%$ restante mujeres. Con respecto a la escolaridad, el $67.6 \%$ de los adolescentes se encontraban en escuela secundaria, un $22.1 \%$ se encontraba en escuela primaria, un $7.4 \%$ atendían una escuela secundaria especial y solo el $3 \%$ de los participantes cursaban educación superior terciaria o universitaria. En cuanto al lugar de procedencia, un 73,5\% de la muestra residía en provincia de Buenos Aires, un $17,6 \%$ en Ciudad Autónoma de Buenos Aires y un $8,8 \%$ en otra provincia de Argentina.

\section{Técnicas para la recolección de datos}

El protocolo administrado consistió en un consentimiento informado, un cuestionario de datos socio-demográficos, y los instrumentos Genetic Health Literacy Screening Tool, y Psychological Adaptation Scale.

Los datos socio-demográficos y familiares relevados fueron: nivel de instrucción, situación laboral, estado civil, cantidad de hijos, cobertura social.

Los niveles de alfabetización en genética fueron evaluados a través de la escala Genetic Health Literacy Screening Tool REAL-G-Sp ${ }^{18}$. Esta escala incluye una tarea de reconocimiento de 62 palabras (puntaje entre 0 y 62), considerando como nivel adecuado de alfabetización cuando la persona puede reconocer al menos 59 términos $^{29}$. Una segunda actividad consiste en indicar por un lado el grado de familiaridad con 8 vocablos (puntaje entre 1 y 7 ): Genética, Cromosoma, Susceptibilidad, Mutación, Variación, Anormalidad, Herencia y Esporádico ${ }^{30}$.

Por otro lado, debían responder un cuestionario, con formato de Elección Múltiple o múltiple choice para ver sus niveles de comprensión (puntaje entre 0 y 8) de estos mismos términos17. Por último incluimos una medida de aritmética (puntaje entre 0 y 3 ) que hace referencia a las habilidades de la persona para procesar conceptos numéricos y de probabilidad básicos ${ }^{31}$.

El nivel de adaptación psicológica a la enfermedad genética fue medido a través de la escala Psychological Adaptation Scale (PAS). Esta escala fue diseñada para ver la extensión de la adaptación en un momento determinado ${ }^{24}$. Está compuesta por 15 ítems con opciones de respuesta tipo likert que componen cuatro sub-escalas, cada una de las cuales representan un dominio del concepto de adaptación, siendo estos: Afrontamiento eficaz, Autoestima, Bienestar espiritual o existencial, e Integración 
social. La escala puntúa del 1 al 5 , donde a mayor puntaje, mayor nivel de adaptación.

\section{Análisis de datos}

El análisis de datos se realizó a través del SPSS (Statistical Package for the Social Sciences)Statistics-25. Con el propósito de describir los niveles de adaptación psicológica y de alfabetización genética se calcularon las medias y desvíos estándar de las dimensiones de estas escalas. Se revisó la normalidad de la muestra por el método Kolmogorov-Smirnov y al no tener una distribución normal se optó por usar el estadístico no paramétrico de Rho de Spearman para estudiar la correlación entre las variables.

\section{Aspectos éticos}

Solo participaron del estudio aquellos adolescentes que además de dar su asentimiento, contaran con la autorización y firma de sus padres en el consentimiento informado donde se explicaba previamente a la evaluación, el objetivo del presente estudio y sus derechos de participación en el mismo. Los datos personales de identificación se mantuvieron en forma confidencial mediante la codificación de los cuestionarios según la Ley Nacional 25.326 de Protección de Datos (Habeas Data).

\section{Resultados}

El primer objetivo de la investigación era conocer el grado de alfabetización en genética en sus cuatro componentes principales. En cuanto al reconocimiento de términos utilizados en la consulta genética, se considera un nivel adecuado de alfabetización cuando se reconocen más de 58 palabras de las presentadas. Se encontró una media de 54,59 palabras reconocidas ( $D E=8,57$ ). Sólo un $39,7 \%$ de los adolescentes que asistían al Servicio de Genética presentaban un nivel adecuado de alfabetización genética. En cuanto a la familiaridad con 8 de los términos más utilizados en el asesoramiento genético, las medias más altas se encontraron en los términos "herencia" ( $M=5,37$, $\mathrm{DE}=1,9)$ y "anormalidad" ( $\mathrm{ME}=4,54, \mathrm{DE}=2,22)$, y las más bajas en los términos "esporádico" $(M=2,33, D E=1,79)$ y "susceptibilidad" (M=2,59, $D E=1,93)$. En relación a la comprensión de estos 8 términos, se encontró una $M=4,06$ $(\mathrm{DE}=2,09)$. Por último, se halló una media de 0,71 $(\mathrm{DE}=0,9)$ para el componente de aritmética.

El segundo objetivo consistió en describir los niveles de adaptación psicológica. Los resultados indicaron una $M=4,02(D E=0.8)$ para la escala general de adaptación psicológica. A su vez, se encontraron medias elevadas para todas las dimensiones de la Psychological Adaptation Scale (PAS) (Tabla 1).

Tabla $N^{\circ}$ 1: Análisis descriptivo dimensiones Psychological Adaptation Scale

\begin{tabular}{lll}
\hline \multicolumn{1}{c}{ Dimensiones } & Media & DE \\
\hline Afrontamiento & 3,82 & 0,93 \\
Autoestima & 4,08 & 0,95 \\
Integración social & 4,16 & 1,11 \\
Bienestar Espiritual & 4,05 & 1,26 \\
\hline
\end{tabular}

Por último, se estudió si existía relación entre las variables mencionadas. Según los resultados hallados no se encontraron relaciones estadísticamente significativas entre los niveles de adaptación psicológica y los niveles de alfabetización genética. Sin embargo, si se encontraron relaciones significativas, moderadas y directamente proporcionales, entre los niveles de alfabetización genética (reconocimiento y comprensión) y los niveles de escolaridad tanto del adolescente como de la madre $(r S=0,314, p=0.015$; $r S=0,283, p=0.03 ; \quad r S=0,352, p=0.007$ ), es decir que a mayores años de escolaridad mejores serían los niveles de alfabetización en genética.

\section{Discusión}

El estudio realizado es un aporte original que permite un mayor entendimiento de la población de adolescentes que acuden a un asesoramiento genético. El nivel de alfabetización en genética que tienen los propios adolescentes, que acuden al servicio de genética, no había sido investigado anteriormente y representa un área de interés.

Según los resultados obtenidos, los niveles de alfabetización en genética de los adolescentes que acuden a un servicio de genética parecerían ser bajos, dado que menos de la mitad de los participantes reconocen de manera correcta los términos que Erby et al. ${ }^{29}$ consideran de uso frecuente en el asesoramiento genético. En cuanto a la familiaridad con los términos que propone Hooker et al. ${ }^{16}$ los adolescentes parecen presentar menor familiaridad con los términos "esporádico" y "susceptibilidad" y mayor familiaridad con "herencia" y "anormalidad". Si bien parecen estar familiarizados con estos términos genéticos, los niveles de comprensión de los mismos son bajos. Estos resultados coinciden con estudios anteriores en población clínica que plantean que si bien los pacientes o sus cuidadores reconocen los términos genéticos, tienen dificultades para comprender el significado real de los mismos y la relación que existe entre ellos ${ }^{19-20}$. De igual forma, determinamos niveles muy bajos en las tareas de aritmética propuesta por Schwartz ${ }^{30}$ es decir que los adolescentes no comprenden los conceptos numéricos necesarios para comprender las nociones de probabilidad involucrados en el origen y herencia de su enfermedad. Esta dificultad en el manejo de las probabilidades representa una alerta ya la aritmética es fundamental para la comunicación del riesgo y la toma de decisiones futuras del paciente ${ }^{17}$.

La comprensión de la información genética, sobre todo en cuanto a la herencia de la enfermedad, vendría a ser uno de los objetivos principales del Asesoramiento genético en esta población, ayudando a los adolescentes a tomar decisiones informadas en relación a la planificación familiar. Siendo que las características del presente estudio comprendían sujetos que contaban con bajos niveles de educación, los resultados obtenidos permiten pensar a futuro en la necesidad de ofrecer a esta población mejores herramientas psico-educativas, para ayudar en la mejor comprensión de nociones de genética, principalmente cuando el nivel de educación es bajo.

En segundo lugar, se encontraron buenos niveles de adaptación en todas las dimensiones de la adaptación psicológica. Los niveles más elevados estaban en la dimensión de integración social, en consonancia con 
estudios previos que señalan la importancia que juega el apoyo social y las relaciones interpersonales en la adaptación psicológica ${ }^{25}$.Por otro lado, si bien los puntajes más bajos pertenecen a la dimensión de afrontamiento eficaz, estos niveles siguen mostrando una adaptación psicológica adecuada. Estos resultados coinciden con lo planteado por Abbott y colaborares ${ }^{27}$.

Los resultados globales mostraron que los adolescentes que acuden al servicio de genética, de población Argentina, están logrando adaptarse psicológicamente a la enfermedad, a pesar del estrés que ésta les genera en los distintos aspectos de su vida. Estos resultados coinciden con otros autores que plantean que la mayoría de las familias logran adaptarse a la enfermedad ${ }^{5,25}$, y con un estudio realizado en adolescentes con Síndrome de Klinefelter que demostró buenos niveles de adaptación psicológica en los mismos ${ }^{28}$.

Por último, si bien no se encontró una relación estadísticamente significativa entre los niveles de adaptación psicológica y los niveles de alfabetización genética, sí se encontraron relaciones significativas entre la alfabetización genética (reconocimiento y comprensión) y los niveles de escolaridad tanto del adolescente como de la madre. Es decir que aquellos adolescentes y madres que tienen más años de escolaridad serían los que tienen un mayor y mejor entendimiento de términos de genética médica. No se encontraran antecedentes que estudiaran la relación entre estas variables.

Las conclusiones de la presente investigación indican: -Los adolescentes diagnosticados con enfermedades genéticas en Argentina poseen un buen nivel de adaptación psicológica a su enfermedad.

-Los adolescentes diagnosticados con enfermedades genéticas en Argentina poseen bajos niveles de alfabetización genética.

-La adaptación psicológica y la alfabetización genética parecen no estar relacionadas entre sí.

-Los niveles de alfabetización genética de los adolescentes diagnosticados con enfermedades genéticas parecen estar relacionados a los años de escolaridad tanto de los adolescentes como de sus madres.

Limitaciones de responsabilidad:

La responsabilidad del trabajo es solo de los autores.

Fuentes de apoyo:

Esta investigación fue realizada en el Centro de Investigaciones de Psicología y Psicopedagogía de la Pontificia Universidad Católica Argentina, en el marco de una Beca de Iniciación a la investigación.

\section{Originalidad:}

Este artículo es original y no ha sido enviado para su publicación a otro medio de difusión científica en forma completa ni parcialmente.

\section{Cesión de derechos:}

Los participantes de este trabajo ceden el derecho de autor a la Universidad Nacional de Córdoba para publicar en la Revista de la Facultad de Ciencias Médicas y realizar las traducciones necesarias al idioma inglés.

\section{Conflicto de interés:}

No se reporta conflicto de intereses.

\section{Agradecimiento:}

Se agradece a los Profesionales del Servicio de Genética Médica del Hospital de Niños Dr. Ricardo Gutiérrez que brindaron su espacio y tiempo para el desarrollo de la investigación. A todos los adolescentes y sus familias que generosa y desinteresadamente han participado del estudio.

\section{Bibliografía}

1.von der Lippe C, Diesen PS, Feragen KB. Living with a rare disorder: a systematic review of the qualitative literature. Mol Genet Genomic Med. 2017 Nov;5(6):758-773. doi: 10.1002/mgg3.315..

2. Quittner AL, Goldbeck L, Abbott J, Duff A, Lambrecht P, Solé A, Tibosch MM, Bergsten Brucefors A, Yüksel $H$, Catastini $P$, Blackwell L, Barker D. Prevalence of depression and anxiety in patients with cystic fibrosis and parent caregivers: results of The International Depression Epidemiological Study across nine countries. Thorax. 2014 Dec;69(12):1090-7. doi: 10.1136/thoraxjnl-2014-205983.

3. Federación Argentina de Enfermedades Poco Frecuentes. Informe Las Enfermedades Poco Frecuentes en Argentina. 2018 Feb; Recuperado el 02 de junio del 2019 de http://fadepof.org.ar/noticias/219.

4.Witt MM, Jankowska KA. Breaking bad news in genetic counseling-problems and communication tools. J Appl Genet. 2018 Nov;59(4):449-452. doi: 10.1007/s13353-018-0469-y.

5. Biesecker BB, Erby L. Adaptation to living with a genetic condition or risk: a mini-review. Clin Genet. 2008 Nov;74(5):401-7. doi: 10.1111/j.1399-0004.2008.01088.x.

6. Austin J, Semaka A, Hadjipavlou G. Conceptualizing genetic counseling as psychotherapy in the era of genomic medicine. $J$ Genet Couns. 2014 Dec;23(6):903-9. doi: 10.1007/s10897-0149728-1

7.Valentine, F., \& Lowes, L. Nursing care of children and young people with chronic illness. Blackwell Publishing Ltd. 2007. doi: 10.1002/9780470692103.

8. Shiloh, S. Genetic counseling: A developing area of interest for psychologists. Prof Psychol Res Pr. 1996;27(5):475-86. doi: 10.1037/0735-7028.27.5.475

9. Vásquez Cerdas, M., Cuenca Berger, P., Campos Ramírez, D., Gutiérrez Doña, B., Fernández Morales, H., \& Morales Montero, F. Abordaje integral de pacientes costarricenses afectados con la enfermedad de Huntington y sus familiares. Acta Med Costarric, 2011;53(3):136-43.

10. McCauley E, Feuillan P, Kushner H, Ross JL. Psychosocial development in adolescents with Turner syndrome. J Dev Behav Pediatr. 2001 Dec;22(6):360-5. doi: 10.1097/00004703200112000-00003.

11. Girón, R.M., \& Cuadrado, F. Aspectos psicológicos del paciente con fibrosis quística: ¿qué ocurre cuando la enfermedad avanza? Rev Patol Respir. 2006;9(2)53-4

12. Lumley MA, Jordan M, Rubenstein R, Tsipouras $P$, Evans MI. Psychosocial functioning in the Ehlers-Danlos syndrome. Am J Med Genet. 1994 Nov 1;53(2):149-52. doi: 10.1002/ajmg.1320530206.

13. Higham L, Ahmed S, Ahmed M. Hoping to live a "normal" life whilst living with unpredictable health and fear of death: impact of cystic fibrosis on young adults. J Genet Couns. 2013 Jun;22(3):374-83. doi: 10.1007/s10897-012-9555-1.

14. Taylor RM, Gibson F, Franck LS. The experience of living with a chronic illness during adolescence: a critical review of the literature. J Clin Nurs. 2008 Dec;17(23):3083-91. doi: 10.1111/j. 1365-2702.2008.02629.x.

15. Bowling BV, Acra EE, Wang L, Myers MF, Dean GE, Markle GC, Moskalik CL, Huether CA. Development and evaluation of a genetics literacy assessment instrument for undergraduates. Genetics. $2008 \quad$ Jan;178(1):15-22. doi: 10.1534/genetics.107.079533.

16. Hooker GW, Ormond KE, Sweet K, Biesecker BB. Teaching genomic counseling: preparing the genetic counseling workforce for the genomic era. J Genet Couns. 2014 Aug;23(4):445-51. doi: 10.1007/s10897-014-9689-4

17. Lea $D H$, Kaphingst KA, Bowen D, Lipkus I, Hadley DW. Communicating genetic and genomic information: health literacy and numeracy considerations. Public Health Genomics. 2011;14(4-5):279-89. doi: 10.1159/000294191. 
18. Rodríguez SA, Roter DL, Castillo-Salgado C, Hooker GW, Erby $\mathrm{LH}$. Translation and validation of a Spanish-language genetic health literacy screening tool. Health Psychol. 2015 Feb;34(2):120-9. doi: 10.1037/hea0000162.

19. Emery J, Kumar S, Smith H. Patient understanding of genetic principles and their expectations of genetic services within the NHS: a qualitative study. Community Genet. 1998;1(2):78-83. doi: 10.1159/000016141.

20. Solomon G, Greenberg J, Futter M, Vivian L, Penn C. Understanding of genetic inheritance among Xhosa-speaking caretakers of children with hemophilia. J Genet Couns. 2012 Oct;21(5):726-40. doi: 10.1007/s10897-012-9495-9

21. Calsbeek H, Morren M, Bensing J, Rijken M. Knowledge and attitudes towards genetic testing: a two year follow-up study in patients with asthma, diabetes mellitus and cardiovascular disease. J Genet Couns. 2007 Aug;16(4):493-504. doi: 10.1007/s10897-006-9085-9.

22. Cheuk DK, Wong SM, Choi YP, Chau AK, Cheung YF. Parents' understanding of their child's congenital heart disease. Heart. 2004 Apr;90(4):435-9. doi: 10.1136/hrt.2003.014092.

23. Fitzgerald-Butt SM, Klima J, Kelleher K, Chisolm D, McBride KL. Genetic knowledge and attitudes of parents of children with congenital heart defects. Am J Med Genet A. 2014 Dec;164A(12):3069-75. doi: 10.1002/ajmg.a.36763.

24. Biesecker BB, Erby LH, Woolford S, Adcock JY, Cohen JS, Lamb A, Lewis KV, Truitt M, Turriff A, Reeve BB. Development and validation of the Psychological Adaptation Scale (PAS): use in six studies of adaptation to a health condition or risk. Patient Educ Couns. 2013 Nov;93(2):248-54. doi: 10.1016/j.pec.2013.05.006.

25. Barakat LP, Linney JA. Children with physical handicaps and their mothers: the interrelation of social support, maternal adjustment, and child adjustment. J Pediatr Psychol. 1992 Dec;17(6):725-39. doi: 10.1093/jpepsy/17.6.725.

26. Jangra D, Ganesh A, Thackray R, Austin L, Ulster A, Sutherland J, Levin AV. Psychosocial adjustment to visual loss in patients with retinitis pigmentosa. Ophthalmic Genet. 2007 Mar;28(1):2530. doi: 10.1080/13816810701201930.

27. Abbott J, Hart A, Morton A, Gee L, Conway S. Health-related quality of life in adults with cystic fibrosis: the role of coping. $J$ Psychosom Res. 2008 Feb;64(2):149-57. doi: 10.1016/j.jpsychores.2007.08.017.

28. Turriff A, Levy HP, Biesecker B. Factors associated with adaptation to Klinefelter syndrome: the experience of adolescents and adults. Patient Educ Couns. 2015 Jan;98(1):90-5. doi: 10.1016/j.pec.2014.08.012.

29. Erby $L H$, Roter D, Larson S, Cho J. The rapid estimate of adult literacy in genetics (REAL-G): a means to assess literacy deficits in the context of genetics. Am J Med Genet A. 2008 Jan 15;146A(2):174-81. doi: 10.1002/ajmg.a.32068.

30. Schwartz LM, Woloshin S, Black WC, Welch HG. The role of numeracy in understanding the benefit of screening mammography. Ann Intern Med. 1997 Dec 1;127(11):966-72. doi: 10.7326/0003-4819-127-11-199712010-00003. 